\title{
La escuela en tiempos de crisis: Puntos de fuga para re-instaurar la esperanza en contextos post-desastre
}

\author{
The school in times of crisis: \\ Projecting points to restore hope in post disaster \\ contexts
}

Bibiana Vélez Medina ${ }^{1}$

Resumen

La modernidad contingente es el resultado de la modernidad ilustrada e industrializada, de allí que la contingencia devenga en riesgo y se instale como condición de posibilidad contemporánea. El riesgo aparece entonces como un constructo social e histórico que permea todos los ámbitos de la cultura occidental, entre ellos el de la educación, el cual debe ser deconstruido desde la emergencia de la incertidumbre que produce la catástrofe del ideal moderno. En este orden de ideas, el presente texto indaga sobre las nuevas condiciones de oportunidad de la escuela en el contexto post-desastre y los riesgos del futuro devenir educativo.

\section{Palabras clave:}

Riesgo, escuela, crisis, incertidumbre, desastre.

\section{Abstract}

Contingent modernity can be described as the result of enlightened and industrialized modernity and hence bears the risk of being transformed into a condition of contemporary possibility. In this manner, risk appears as a social and historical construct permeating all fields of western culture, among them the field of education, which must be deconstructed from the emergency of uncertainty, which produces the catastrophe of the modern ideal. From this vein of ideas, the following text examines new conditions of opportunity for the school in a post-disaster context and the risks of future educational transformation.

Keywords:

Risk, school, uncertainty, disaster, crisis.

Artículo recibido el 17 de mayo de 2011 y aprobado el 19 de agosto de 2011

1 Vicerrectora Académica de la Universidad La Gran Colombia - Seccional Armenia. Líder del Grupo de Investigación: PAIDEIA (Categoría A - Colciencias). Correos electrónicos: viceacad@ugca.edu.co; bibiambar@hotmail.com. 


\section{Introducción}

Ligada a la noción de incertidumbre, la idea del riesgo reaparece indiscriminada y aleatoria, una y otra vez, en el tiempo presente. Su concreción no es otra cosa que la manifestación de un temor previsto o imprevisto, la exposición a nuestros límites de seguridad, la posibilidad de quedar aniquilados ante el asalto de las propias certezas o la opción de emerger hacia nuevos horizontes.

En efecto, basta con dar una mirada a los eventos recientes que han golpeado la estabilidad y fijeza de naciones enteras (Japon, 2011; Haití, 2010; Chile, 2010) o con recordar catátrofes tan cercanas que en definitiva, derrumbaron cualquier parámetro de seguridad (terremoto en el Eje Cafetero, 1999) para entender que los desastres naturales son una realidad contingente para la humanidad de todos los siglos.

Asistimos impávidos al escenario de las peores catástrofes de la historia, que en lugar de reducirse debido al supuesto avance de la ciencia, están generando mayores desastres en razón de la intervención fragmentada del hombre sobre la naturaleza en aras del progreso, lo cual, sumado a la concentración urbana y a la organización territorial de las ciudades en el mundo actual (Mitchell, 1994), nos sitúa frente a la naturaleza de un riesgo que aún no se ha comprendido ni dimensionado desde las lógicas cartesianas que dificultan concebir al hombre en interrelación con el planeta.

Cuando los grandes desastres destruyen las edificaciones, arrasan con las vidas de los cercanos, dejan sin hogar a innumerables familias y alteran para siempre el devenir rutinario de un contexto, la escuela no puede voltear la mirada ignorando como acostumbra- los sucesos de una sociedad en crisis. El Sistema Educativo Latinoamericano, reconocido por sus grandes problemáticas, está llamado en medio del desastre a revestirse de nuevas formas, superar sus desaciertos y enfrentar con pertinencia los contextos que demandan una voz de esperanza, una estrategia inteligente y una fuerza colegiada, un nosotros concertado para poder re-construirse, ya no desde los parámetros tecno-burocratizados y desgastados de los muros del aula o de los tiempos controlados del currículo, sino desde la ciudad, la vida y la otredad como esenario de encuentro no escolarizado para el aprendizaje.

El presente texto es una propuesta embrionaria de indagación, para la cual las nociones capitales de "otredades", "reforma del pensamiento educativo" y "escenarios de ciudad como espacio educativo", son ejes que atraviesan y cautivan la presente idea investigativa.

\section{Argumentos iniciales: la naturaleza de la escuela ${ }^{2}$}

La escuela es quizá el proyecto más representativo y viabilizador de las sociedades modernas. Si bien, como lo afirma Terrén $(1999$, p. 67), la escuela no fue un invento de la modernidad ilustrada, «sí lo fue su configuración como organización de masas y como principal anatematizador de la ociocidad»; fue concebida y estructurada para instaurarse como estamento formal, en donde se privilegia la perpetuación y legitimación de los bienes y sentidos de la humanidad, así como para difundir la red de significados o «el magma de las significaciones imaginario sociales, las cuales son llevadas por la sociedad e incorporadas a ella y, por así decirlo, la animan» (Castoriadis, 1986).

La idea moderna sustentada en torno a que «al igual que no hay progreso sin productividad, no hay tampoco productividad sin disciplina» (Terrén, 1999), sentó los fundamentos de la escuela para garantizar la expansión y el ideal de progreso económico, político, social y cultural del mundo occidental.

La escuela se ha convertido, desde entonces, en el símbolo cultural para la institucionalización del conocimiento y para el agenciamiento de los modos sociales en el contexto de una comunidad histórica de maestros y estudiantes.

Desde otra perspectiva, es preciso anotar que la escuela no sólo ejerce una función de «perpetuación de los relatos» (Lyotard, 2000), «reproducción de las

2 Aquí entendida como aquella noción genérica para designar cualquier estamento formal en que se desarrolla un currículo gradual desde la educación preescolar hasta la universitaria. 
estructuras sociales y fabricación de los modos de pensar y actuar de las personas» (Bourdieu, 1991) o «reproducción de los imaginarios» (Castoriadis, 1986), sino que además, y en contraposición a lo anterior, es considerada como el motor para la renovación y movilización de las estructuras viejas que necesitan ser transformadas. En efecto, se debate en la tensión antagónica/complementaria de ser, por un lado, institución determinada que reproduce y conserva las crisis sociales en sus estructuras, pero a su vez, sistema vivo que denuncia la actual devastación humana y proclama el advenimiento de una sociedad en la cual nos situemos incluyentes, renovados, solidarios y realizados.

A partir de Luhmann (2006), se infiere que el sistema histórico-social ha creado y es creado por el sub-sistema educativo, atendiendo a una dinámica auto-referenicial y auto-poiética, en cuyo juego se fraguan en la escuela las expectativas culturales para la conservación y preservación, pero también para la transformación y la renovación del conocimiento, la ciencia y la tecnología. En su naturaleza subyace una dualidad que la circunscribe, pues encierra en sí un juego de contrarios: es a la vez determinada/ indeterminada, simple/compleja y se debate entre la conmemoración de un presente pasado y el anhelo de un presente porvenir.

Sin embargo, a pesar de la posibilidad creadora que suscitan estas tensiones, la escuela tiende hacia la estaticidad de su pensamiento y estructura. La escuela del tiempo presente no escapa a la tendencia natural de evitar la incertidumbre y procurar mantener un estado equilibrado, cierto y ordenado; como todo sistema, intenta mantener su organización y preservar su autonomía. En efecto, la escuela se ha pensado como un estamento de la cultura, se creó para permanecer, ; y por tanto, no está preparada para enfrentar los acontecimientos inesperados de la sociedad-historia, no está diseñada para devenir en un estado de vigilancia conceptual y experiencial, no está capacitada para actuar bajo la ruina de sus inamovibles.

\section{Algunas crisis de la escuela del tiempo presente}

"Supuestamente, la modernidad iba ser aquel periodo de la historia humana en el que, por fin, nos sería posible dejar atrás los temores que dominaron la vida social del pasado, hacernos con el mando de nuestras vidas y dominar las descontroladas fuerzas de los mundos social y natural. $\mathrm{Y}$, sin embargo, volvemos a vivir una época de miedo. Tanto si hablamos del miedo a los desastres naturales y a las catástrofes medioambientales, como del miedo a los atentados terroristas indiscriminados, hoy en día el mundo experimenta un estado de ansiedad constante por los peligros que pueden azotarnos sin previo aviso y en cualquier momento" (Z. Bauman, 2007).

\section{Paradigma determinista: nuestras instituciones aún no han logrado superar la lógica del paradig- ma determinista, simplificado y reduccionista, heredado de la Modernidad Ilustrada. Esto se evidencia en el modo de relación disyuntiva entre las instancias académico-administrativas, en la rigidez de las estructuras de conocimiento- currículos-planes de estudio, en la multiplicidad de procesos y procedimientos aislados de la lógica integradora, en la inminente ausencia de escena- rios de pensamiento dedicados a la crítica radical de nuestras cegueras y errores.}

La academia se esfuerza por conservar y "transmitir" un conocimiento que no logra renovarse, que cada vez es más hiper especializado, estático, compartimentado en campos delimitados del saber, poco sometido a racionalidades críticas y segregado en su esencia del todo sistémico al cual pertenece. Por un camino paralelo, disyuntivo, trasiega la administración, quien en su intento por ser estratégica, planificada y controlada, olvida las reflexiones frente al conocimiento, ignora los puntos de encuentro y retroalimentación con la academia, suscitando en ascenso la descontextualización de sus acciones.

2. Temor a la incertidumbre: al igual que todo ser viviente, el sistema educativo posee sus dispositivos y barreras de autoprotección, defensa, huída, ataque, para la preservación de su equilibrio en 
razón a su tendencia a perdurar. Así, la escuela como sistema, produce y organiza permanentemente una especie de membrana-frontera, de límite excluyente que selecciona y filtra los intercambios con el entorno, permitiendo el paso de lo asimilable a su estructura (lo ya conocido) y oponiéndose a integrar lo no-asimilable (lo desconocido).

3. Crisis de la docencia: "Han entrado, o quizás, permanecido en crisis, los AXIOMAS que sustentan la ética, la praxis, el demos, el ontos de la episteme docente" (Guarín, 2004). Aún no se define con claridad quién es el sujeto docente, cuáles son los criterios que fundamentan la enseñanza, cómo permitir la reintegración entre ciencia y filosofía para encontrar el origen filosófico del conocimiento en su relación interdependiente con la ciencia, cómo atender al llamado de la indagación sostenida, y ante todo, cómo entender que el rol del maestro no es "enseñar-lo-dicho", sino el "estar-diciendo" (Melich, 1998) en un continuo acontecimiento de estar-aprendiendo.

De esta manera, Nietzsche lo anunciaba al referirse a los docentes de su época:

La triste causa de que, a pesar de todo, no consiga manifestarse por ningún lado una honradez completa, es la pobreza espiritual de los profesores de nuestra época: precisamente en ese campo faltan los talentos realmente inventivos, faltan los hombres verdaderamente prácticos, o sea, los que tienen ideas buenas y nuevas, y saben que la auténtica genialidad y la auténtica praxis deben encontrarse necesariamente en el mismo individuo (2000; versión original, 1872).

4. La crisis de los metarrelatos: las grandes teorías explicativas, asentadas sobre la presunción de una verdad determinada para garantizar un progreso ilimitado, ya no nos sirven para comprender el mundo, han caído en descrédito porque perdieron su legitimación y, por tanto, su papel totalizante (Lyotard, 2000). Los discursos viejos, lisiados y desesperanzadores se han tomado la hermenéutica del sentido de humanidad. Los criterios de formación son poco sustentables. Las peroraciones dialécticas continúan separando a sujeto- objeto, y aún no se encuentra el camino para conjugar articuladamente un nuevo metarrelato del conocimiento. Las preguntas por la libertad, la autonomía, la autorealización del sujeto, la emancipación del pensamiento, no han superado los lenguajes que simplifican y anulan la posibilidad de creación para dejar ser, dejar hacer, dejar aprender, dejar pensar... Superar la crisis del relato implica un desafío para comprender las imágenes tecnocientíficas del mundo, para aventurarse en los nuevos juegos lingüísticos que no pasan por el libro, para comprender las connotaciones éticas/ políticas/jurídicas/epistémicas de las nuevas narrativas. Aún la escuela no sabe cómo acompasar los lenguajes contradictorios sin simplificarlos, cómo conjugar arte-literatura-religión-mitolenguaje telemático... Tampoco hemos logrado conjurar un lenguaje que nos acerque al mundo, al contexto, a las realidades de vida de la humanidad en crisis; el lenguaje de la escuela aísla, excluye y discrimina en lugar de generar mayor dialogicidad con la sociedad.

5. «La institucionalización del Autismo» (Sánchez, 2007): nuestro sistema educativo ha desarrollado una especie de inteligencia ciega, ensimismada y cerrada que le impide el desarrollo de una adecuada interacción social, comunicación y reciprocidad con la auto-eco-regulación sistémica que la habita y en que habita. La escuela se ha aislado y la sociedad así lo ha asumido.

6. El aula olvidada: se ha burocratizado tanto el sistema educativo y sobre él han recaído tantas responsabilidades delegadas - la de la familia, el estado, la salud, la iglesia, la ley- que nuestras escuelas de hoy congregan más atenciones y tratamientos que aprendizajes, han perdido su esencia de escenario-encuentro para la formación humana. Los docentes deben llenar incontables formatos; entregar cientos de reportes, informes, estadísticas y datos; asistir a reuniones, capacitaciones y encuentros que ocupan su principal atención, invaden la gran parte de su tiempo y colman su escasa agenda laboral. Por último, escondida tras la inmensa cantidad de actividades que "garantizan la calidad", tímida y olvidada queda la responsabilidad del Aula, relegada y atendida 
después del último informe, reunión y formato. El sentido fundamental de la escuela -el aula, como espacio/tiempo de interacción para conservar y renovar el conocimiento procurando la sabiduría en contexto- se ha ignorado y sencillamente se ha incorporado a la práctica desgastada del maestro.

\section{En medio de la incertidumbre y el colapso, la escuela queda aturdida e inmovilizada}

En el mundo en que estamos viviendo hay muchas cosas que se están deteriorando [...]; nuestra naturaleza está cada vez más deteriorada y agredida, crecen los desiertos, se destruyen selvas extraordinarias e importantes para la supervivencia de tantas especies en el planeta; miles y miles de personas, cientos de miles de personas mueren en presuntos desastres naturales, que por cierto no son de origen natural sino que son de origen humano y en su última manifestación solamente son una expresión de la naturaleza" (Max Neef, 1991).

La idea que prevaleció desde Descartes hasta Newton acerca de un mundo ordenado, cierto, predecible y dominado por la ciencia, se derrumba ante las evidentes situaciones de crisis, incertidumbre y caos que desbordan nuestra posibilidad de control sobre los fenómenos. En efecto, toda idea de dominio del hombre sobre la naturaleza pierde su validez cuando nos golpean las incontenibles e inesperadas crisis económicas, políticas, religiosas, culturales y más aún, ante la ocurrencia de las catástrofes naturales que arrasan con la vida, la estabilidad y la soberanía humana: ¿qué es el hombre frente a la magnitud imponente de un terremoto, un tsunami, una inundación, un huracán o una exposición a la radiactividad? ¿quién está excento de perderlo todo, incluso la vida, en un desastre sufrido por aquella naturaleza que nos sostiene como especie en el planeta? ¿qué queda de lo humano y sus instituciones ${ }^{3}$ después de una catástrofe?

3 En el sentido de Castoriadis, entendidas las instituciones sociales como el conjunto de normas, valores, creencias y bienes culturales que dan sentido a las interpretaciones del mundo en un determinado contexto.
El sistema educativo, estructurado para perdurar y perpetuar, caracterizado por su estática quietud, organizado para garantizar la reproducción de la cultura, incorporado en el quehacer autómata de millones de niños, niñas y jóvenes; sistema seguro, amurallado e inexpugnable, cuando se halla en medio de una devastación que derriba las paredes de sus aulas, sencillamente queda desnudo e impábido por su vulnerabilidad e incapacidad de actuar en y por el desorden.

Resulta importante anotar que el mundo físico se despliega en medio de tracciones entre movimientos contrarios y complementarios "eventos-enatracción” que generan posibilidades de creación, transformación y regeneración de la vida; estos movimientos podrían representarse de la siguiente manera:

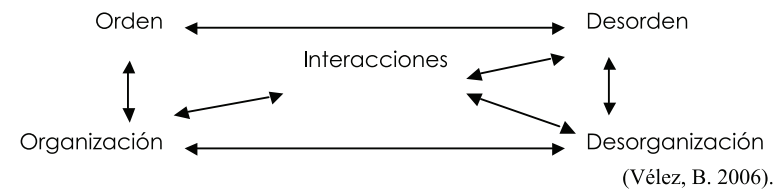

Dichos eventos de atracción-tensión nos recuerdan la latencia constante de lo incierto en el juego impredescible de creación/destrucción, orden/ desorden, organización/desorganización, vida/ muerte, despertando la conciencia por la fragilidad y futilidad de la especie en el planeta.

La idea de fijeza de la escuela queda revaluada en el vértigo producido por las interacciones inciertas y violentas de la naturaleza. Así, cuando el sistema educativo colapsa por las catástrofes, la noción de educación institucionalizada pierde todo su sentido, deja de ser funcional para sobrepasar la crisis, y entonces la sociedad se enfrenta a la re-configuración de su imaginario educativo.

Aturdida y desconcertada por la pérdida y desolación que emergen post-desastre, el sistema educativo se enfrenta al reto enorme de generar condiciones de seguridad, estabilidad, protección y mitigación de los impactos físicos, psicológicos, sociales y económicos. La sociedad colapsada vuelve su mirada a la escuela y le exige ser el dispositivo que garantice la supervivencia, pues ella es la única que 
puede significar esperanza en medio de la muerte 4 . Entonces, se hace evidente que, nuestra escuela está tan determinada por las normas y principios de "estabilidad" que no ha podido encontrar una respuesta dinámica y adecuada al cambio rápido en el cual debe afrontar las transformaciones complejas que se suscitan con los desastres.

\section{Los contextos de desastre}

«El ser humano se ha enfrentado desde siempre a la incertidumbre del futuro»

Luhmann, 2006.

«Tal vez nuestras normas y valores culturales no son adecuados; es posible que muchos de nosotros busquemos eliminar el riesgo ambiental completamente o tratemos de apartarnos de él, más que aprender a ajustarnos a sus límites»

Mitchell, 1994.

Podríamos afirmar, a luz de la teoría de Mitchell (1994), que los desastres son problemas todavía no mitigados por el desarrollo de las sociedades contemporáneas, y más aún, que ellos son el resultado de la intervención fragmentada de la inteligencia del hombre sobre la naturaleza.

La problemática del desastre natural atraviesa por la incapacidad del hombre para comprender los fenómenos naturales de una manera sistémica, y esto mismo hace que, desde su inteligencia disyuntiva, no logre intervenir la naturaleza de una forma compleja, generando así mayores desastres colaterales.

4 La Red Interagencial de Educación en Situaciones de Emergencia (INEE, 2010) afirma que "la educación mitiga el impacto psicosocial de los conflictos y desastres, dando una sensación de normalidad, estabilidad, estructura y esperanza para el futuro». Además plantea que, "cuando un niño está en un ambiente de aprendizaje seguro, él o ella tiene menos probabilidades de ser sexual o económicamente explotado o expuesto a otros riesgos, tales como la trata de personas o unirse a un grupo de delincuencia organizada. Además, la educación puede transmitir información vital para fortalecer las habilidades críticas de supervivencia y mecanismos de adaptación, por ejemplo, cómo evitar las minas terrestres, cómo protegerse contra el abuso sexual, cómo prevenir el VIH/SIDA y cómo acceder a la asistencia sanitaria y a la distribución de alimentos. La educación en emergencias también proporciona protección cognitiva, apoyando el desarrollo intelectual a través de la enseñanza de la alfabetización, la aritmética y habilidades de estudio. También puede enseñar a construir la paz y la resolución de conflictos. Puede proporcionar elementos esenciales para la estabilidad económica futura».
Es suficiente con revisar las agendas de planificación post-desastre para concluir que la búsqueda desesperada por encontrar una nueva estabilidad después del caos generado tras una catástrofe natural, es orientada por actuaciones particulares y segmentadas que olvidan o ignoran los tejidos sistémicos en los cuales estamos inmersos. Estas mediaciones fragmentadas hacen que prevalezca la lógica reduccionista y simplificada que no logra dar respuesta integral a fenómenos que son de naturaleza interrelacional.

Por otro lado, no ha sido posible lograr la comprensión profunda del balance que debe existir en el tratamiento de los vínculos entre el fenómeno del desastre y los contextos del desastre. En efecto, se otorga demasiada atención al fenómeno del desastre y se pasan por alto -o se exploran muy poco- los contextos sociales, culturales, políticos y económicos en que ocurren (Mitchell, 1994).

El afán inmediato de los organismos que intervienen post-desastre se centra en la atención humanitaria, posteriormente en la rehabilitación $y$, finalmente, en la reparación. Luego el desarrollo de estas fases ocurre desde miradas externas, planes paternalistas y estrategias inmediatistas que ignoran los contextos y se enfocan en el fenómeno.

Dicha simplificación nos lleva a olvidar o desconocer que el contexto social, histórico, económico, político, educativo y cultural en que ocurren las catástrofes condiciona las posibilidades y potencialidades de atención e intervención. Así se ignora la constricción y, a su vez, la emergencia creadora en la interacción desastre/contexto.

Pero no sólo se descontextualiza el fenómeno del lugar específico y de sus contextos sistémicos de ocurrencia, sino que además las explicaciones actuales sobre los desastres no dan un peso adecuado a la acción forzada y sin precedentes de los cambios sociales y ambientales contemporáneos (Mitchell, 1994). Por consiguiente, es posible afirmar que la desconexión compleja ocurre en tres niveles de ignorancia acerca de los contextos que no sólo rodean, sino que condicionan/posibilitan la reconfiguración socio-histórica. Estos son: 


\section{Contextos espaciales: el "lugar" perdido}

$\mathrm{El}$ análisis del contexto debe ocuparse de la comprensión del lugar donde ocurren los desastres, entendida aquí la comprensión no en términos de acumulación de información o conocimiento, sino como la implicación física, emocional, cognitiva y comunicativa del que conoce, en el objeto de conocimiento, para asumir postura política y ética frente a lo que comprende.

En este sentido, la "comprensión del lugar" no es el simple conocimiento de una localización geográfica en donde ha ocurrido un desastre, sino la comprensión de una entidad que es producto de la interacción humana en el medio ambiente local y que parte de la identidad de sus habitantes, como componente central que ayuda a entender la actitud y el comportamiento de la gente que está en contacto con dicho lugar (Mitchell, 1994). De tal manera que el análisis del contexto espacial debe proporcionar una comprensión profunda de las amenazas y potencialidades enraizadas que emergen tras la catástrofe.

No es posible desconocer que en virtud de los espacios se instauran las relaciones sociales, y que además, según Bachelard, la adherencia a esos lugares hace que tomemos la forma de nuestros escondites (2000, p. 45; versión original, 1957). Entonces, tras la destrucción de los espacios, consecuentemente surgen nuevas formas de interacción humana: constrictivas, egoístas, violentas o solidarias, abnegadas, incluyentes, en todo caso, supeditadas a las restricciones, hacinamientos y temporalidades de los lugares adaptados para el resguardo de los sobrevivientes. Relaciones viabilizadas, por supuesto, por el lugar de sentido que se instaure para el aprendizaje de los nuevos modos de asentamiento.

Un lugar configura las relaciones inscritas en el espacio, relaciones también inscritas en la duración de formas espaciales concretadas en el tiempo, para hacerse lugar de identidad, relacional e histórico. En este orden de ideas, un espacio que no puede definirse en la consolidación de identidad, en la perduración de relaciones y en la configuración histórica, será, sin duda, un no-lugar (Augé, 1996). ¿Qué sucede con los lugares de sentido cuando han sido arrasados por un fenómeno natural? ¿Cómo configurar nuevos lugares de identidad que permitan la recuperación de la esperanza en las comunidades que lo han perdido todo y cuyo contexto espacial ha sido removido o quizás borrado del paisaje geográfico? ¿Cuál es la responsabilidad y la capacidad de la escuela para la configuración de nuevos lugares? $\mathrm{O}$ ¿será que tras la tragedia, lo que se instaura es un no-lugar prolongado?...

Los no-lugares son tanto las instalaciones necesarias para la circulación acelerada de personas y bienes (vías rápidas, empalmes de rutas, aeropuertos) como los medios de trasporte mismos o los grandes centros comerciales, o también los campos de tránsito prolongado donde se estacionan los refugiados del planeta (Augé, 1996, p. 41).

«El no lugar es lo contrario de la utopía: existe y no postula ninguna sociedad orgánica» (Augé, 1996, p. 41). Entre tanto, el espacio es la sublimación del lugar, constituye su evocación, la cristalización en la memoria mediante el recorrido de significados del ser humano que halla en el lugar la transformación en espacio personal de vida. «El espacio sería al lugar lo que se vuelve la palabra cuando es hablada, es decir, cuando está atrapada en la ambigüedad de una ejecución» (Merleau-Ponty, citado por Augé, 1996, p. 85).

Una vez perdido el espacio, las personas quedan expuestas al "afuera", expulsadas -en el sentido de Bachelard (2000) - de la concreción de lo de dentro y arrojadas a la vastedad de lo de fuera: «lo de dentro y lo de fuera no reciben de igual manera los calificativos, esos calificativos que son la medida de nuestra adhesión a las cosas» (p. 188).

Si la escuela no logra generar memoria, realización, espacio personal de vida, sociedad antropobio-crono-lógica, el contexto espacial destruido no será espacio, no será de nuevo lugar, y sus habitantes quedarán condenados al hastío o a la costumbre de permanecer en un tránsito indefinido por un nolugar, en el cual se perdieron los sentidos de vida compartidos, donde se cristaliza el simbolismo de la antropología de la sobremodernidad: sujetohumanidad-tiempo, marchan tan de prisa, que en 
su afán difícilmente logran la acogida cálida de la posibilidad figurativa de hallarse lugar para conjugar identidad y relación, para alcanzar, al menos, una estabilidad mínima, un lugar para la memoria.

\section{Contextos temporales: el desastre como umbral histórico de la potencia}

¿Cuáles son las condiciones para convertir el desastre en posibilidad de ruptura histórica y en movimiento para atravesar los umbrales de la utopía?

Si bien los estudios se han centrado en el análisis geo-referencial de los espacios en condición de desastre, vale la pena preguntarse por el análisis del contexto histórico en que ellos ocurren desde la axialidad temporal. ¿Cuál es la lectura del tiempo presente en los contextos de mundo? y ¿de qué manera influyen sobre la condición post-desastre?

Además, si la sociedad se mantiene unida y perdura en su condición histórica por la suma de normas, valores, lenguaje, instrumentos, procedimientos, métodos que dan sentido particular al Yo individual y colectivo con su tipo y su forma (Castoriadis, 1986), y así mismo se transforma como institución social por dos vías posibles [1. «Superficialmente por la coerción, la violencia, el poder, la ley impuesta y las sanciones, pero 2. Ampliamente por la adhesión, el consenso, la creencia y la legitimación» (Castoriadis, 1986)], podríamos asumir entonces que una situación de catástrofe natural estaría ubicada en ambas vías: la de violencia natural y la del posterior consenso, a partir de las cuales se puede generar una potencia utópica o un pesimismo trágico postdesastre. O bien, surgiría un sentido renovado para hacer una ruptura emancipatoria que de paso a la instauración de nuevas normas, valores, lenguajes e instrumentos sociales para el rescate de una humanidad posible, unida por su necesidad de supervivencia como especie; o en su defecto, la sociedad se transformaría hacia estadios más violentos, primarios, egocéntricos y auto-destructivos.

En este escenario de posibilidad tiene lugar la escuela como institución que puede generar sentidos compartidos, convicciones, adhesiones, fe y estrategia colegiada para romper con las viejas formas de organización y propiciar la instauración de nuevos escenarios de transformación en la sociedad.

Sin embargo, al proponer que el sistema educativo se convierta en punto de fuga para las tensiones entre lo utópico y lo trágico de la catástrofe, tenemos que volver a la pregunta ¿está preparada la escuela para configurar un presente/porvenir en horizonte de esperanza?

\section{Contextos discursivos e imaginarios}

El tercer contexto que debe ser estudiado y comprendido es aquel intangible, imaginario y compartido que hace referencia al conjunto de significaciones imaginarias que dan sentido y otorgan determinados valores a las cosas y a las relaciones en la sociedad.

Un contexto imaginario se sostiene por los relatos, discursos y lenguajes, los cuales, a su vez, son determinados por el paradigma de mundo instaurado en el suprasistema social:

En realidad sería superficial e insuficiente decir que toda sociedad tiene en sí misma un sistema de interpretación del mundo. La sociedad es un sistema de interpretación del mundo; aunque el término "interpretación" es aquí otra vez superficial e inapropiado. La sociedad es una construcción, una constitución, una creación del mundo, de su propio mundo. Su identidad no es sino este sistema de interpretación, este mundo que ella crea. Y a eso se debe que la sociedad sienta (de la misma manera que un individuo) como una amenaza mortal cualquier ataque que se haga contra su sistema de interpretación; tal ataque lo siente contra su identidad, contra sí misma (Castoriadis, 1986).

Teniendo en cuenta lo anterior, el significado y valor de las pérdidas post-desastre serán diferentes para los distintos contextos imaginarios. De igual manera, las urgencias de re-construcción serán variables y supeditadas a las prioridades de una sociedad determinada y auto-organizada por la comunicación, que a su vez es producida y producente de los modos sociales (Luhmann, 2006). ¿Cuál es el valor no-cuantitativo de la pérdida post-desastre? ¿cuál es la organización funcional y la estructura de relaciones que colapsa con la catástrofe? ¿qué 
es lo que la escuela debe ayudar a restaurar a partir de la comprensión de los imaginarios y sistemas de interpretación de mundo del contexto imaginario y discursivo? ¿cuáles son los lenguajes que posibilitan y los que imposibilitan la instauración de una esperanza post-desastre?

\section{La crisis del desastre como oportunidad para la de-construcción del pensamiento educativo tradicional}

En esta guerra, nuestro ganado ha sido tomado por las milicias Janjaweed y tuvimos que huir de nuestra tierra. Tuvimos que dejar atrás todas nuestras pertenencias. Lo único que podría traer con nosotros es lo que tenemos en nuestras cabezas, lo que se nos ha enseñado-nuestra educación. La educación es lo único que no puede ser tomado de nosotros y sobre la cual podemos construir una vida mejor para nuestros hijos. (Las mujeres en el campamento de refugiados de Breijing, al este de Chad, 2005).

En el marco de las condiciones normales de vida, la escuela nos recuerda que la educación es un fenómeno necesario en la vida del ser humano y a la vez un proceso que nos acompaña desde la concepción hasta el final de la existencia. Ante las situaciones de desastre, en las cuales la normalidad se torna en plena anormalidad, desequilibrio y caos, la escuela está llamada a adquirir otras dimensiones que le permitan movilizar sus estructuras y movilizar la re-construcción de las urdimbres sociales. En este sentido, cobran fuerza interrogantes como: ¿Qué entendemos por escuela? ¿Qué podemos esperar de ella para que nos permita vivir mejor? ¿Puede la escuela aprovechar la dificultad para transformarla en oportunidad y para encontrar un nuevo orden/ organización en medio del caos? ¿Es capaz de reconfigurar sus lenguajes para transformar los sentidos viejos en nuevas significaciones de humanidad?

De-construir el imaginario tradicional de escuela es condición prioritaria para re-construir un mundo posible tras el desastre; allí cobra real importancia reconocer que el aprendizaje no está demarcado ni circunscrito a la formalización curricular de la institucionalidad, que se aprende sin necesidad de gradualidad ni relaciones formales o "encierro"; que en efecto se aprende mejor sin la necesidad de un maestro, sin esa «lógica del atontamiento que hace a los alumnos dependientes de la explicación» (Ranciére, 2007), que se aprende y desaprende en un contexto social que instala a los sujetos en el núcleo de múltiples miradas y ámbitos de relación e interacción con sus iguales y con los otros.

La ausencia de una edificación para la educación formal genera una forma distinta de encuentro para el aprendizaje, situado desde la humanidad del otro, desde su profunda sensación y deseo de superación sin la barrera del muro; esto nos permite ser capaces de superar el concepto de escuela como un lugar espacial y temporal para asumirla como un sistema de sentidos, comunicaciones e interacciones de hombres y mujeres que encuentran en el acontecimiento de estar-aprendiendo, "estar-diciendo" (Melich, 1998, p. 97) una disculpa para reconfigurar los modos perdidos que dejó la tragedia. Este marco de posibilidad necesariamente se recorre en el trayecto de una Pedagogía Social que, a la vez, se convierte en eje motor de la reconstrucción de vínculos solidarios, ciudadanos y culturales que van más allá de la simple escolaridad; se trata, ante todo, de una oportunidad para la recuperación de la esperanza colectiva.

Para lograr la configuración de nuevas posibilidades de organización humana post-desastre es necesario que la escuela supere la tensión entre dos polos inmovilizadores del pensamiento: el pesimismo trágico y la utopía ingenua, pues ninguno de estos extremos la sitúa en un plano jurídico de actuación que le permita consolidar apuestas de creación; por el contrario, conduce a los sujetos hacia la indiferencia acrítica, desprovista de una praxis pertinente.

Subyace, entonces, la imperiosa necesidad de atravesar los límites de lo tradicional y propiciar, a raíz del desastre, una modificación profunda de la estructura, funcionamiento y relaciones pedagógicas en las escuelas, y de éstas con la comunidad. 


\section{Ejes de Problematización}

1. ¿Cuál es el valor imaginario de la pérdida postdesastre? ¿Cuál es la organización funcional y la estructura de relaciones que colapsa con la catástrofe?

2. ¿Cuáles son las crisis y posibilidades que revisten a la escuela en el momento de la catástrofe y con las cuales se acompaña para configurar un presente/porvenir tras la ocurrencia de un desastre?

3. ¿Qué queda de la escuela sin la escuela y cómo se configura en el contexto temporal del miedo y en el vacío del lugar? ¿Cuáles son las nuevas connotaciones que se fraguan en el aula sin muros y en el currículo sin tiempos?

4. ¿Qué es lo que la escuela debe ayudar a restituir/ reconstruir a partir de la comprensión de los imaginarios y sistemas de interpretación del mundo en el contexto espacial, temporal y discursivo de una comunidad que se enfrenta a la pérdida? ¿Cuáles son los lenguajes que posibilitan y los que imposibilitan la instauración de una esperanza post-desastre? ¿Es capaz de re-configurar sus lenguajes para transformar los sentidos viejos en nuevas significaciones de humanidad?

5. ¿Cómo superar los modos de intervención disyuntiva que, además de ignorar la complejidad sistémica del lugar, ignoran las relaciones histórico-temporales que afectan e influyen en los contextos de desastre? ¿Es posible la instauración de una lógica transdisciplinaria para la comprensión del fenómeno del desastre y para la re-configuración de una escuela posible?

6. ¿Cuál es el lugar de la memoria y la palabra en la recuperación post-desastre?

7. ¿Cuáles son las condiciones de oportunidad para superar las "lógicas de restitución" de lo perdido y, en lugar de ello, abordar las "lógicas de la reconstrucción" del porvenir?

8. ¿Cuáles son los sentidos de vida y necesidades de aprendizaje que emergen tras la catástrofe?

9. ¿Cómo hallar ángulos de expansión a partir de la tensión entre egocentrismo/abnegación que permitan la prevalencia del nosotros colegiado a partir de la escuela que se levanta post-desastre?
10. ¿Cuál es el pensamiento educativo que fenece con el desastre?

11. ¿Cómo hacer del desastre un punto de fuga para configurar la esperanza de una escuela que trasciende sus crisis y de una sociedad que instaura nuevos modos de pensamiento, lenguaje y actuación? ¿Cuáles son las condiciones para convertir el desastre en posibilidad de ruptura histórica de la escuela y para atravesar los umbrales de la utopía social?

12. ¿Cómo instaurar una geo-política educativa que genere en el sistema escolar capacidad para anticiparse al riesgo y estrategias para atender las catástrofes de manera compleja y pertinente?

\section{Enfoque metodológico}

En principio, se considera que el enfoque más cercano para el abordaje de este interés investigativo sería el histórico-hermenéutico, ya que se cuenta con algunos soportes institucionales realizados desde la labor de los investigadores que integran el grupo de investigación PAIDEIA. Estos soportes son:

1. Propuesta educativa para la atención de emergencias y la re-construcción post-desastres: proyecto enmarcado en la pedagogía social para la inclusión, ganador de la convocatoria ASCUN 2010 y avalado por la misma organización como propuesta de responsabilidad social universitaria.

2. Re-Crear la Escuela, su significado y sentido como contexto vital para la inclusión y la recuperación del sujeto y los modos de intersubjetivación.

3. Vivienda temporal de emergencia para la atención post-desastre: proyecto adelantado por docentes y semilleros de la facultad de Arquitectura, orientado al diseño y construcción de prototipos de vivienda y edificaciones de servicios comunitarios básicos temporales en contextos post-desastre.

4. La escuela busca al niño (1999-2007). Proyecto implementado por el municipio de Armenia post-terremoto del eje cafetero, reconocido y avalado por UNESCO. 


\section{Bibilografía}

Augé, M. (1996). Los no-lugares. Espacios del anonimato. Una antropología de la sobremodernidad. Barcelona: Gedisa S. A.

Bauman, Z. (2007). Miedo líquido: la sociedad contemporánea y sus temores. Barcelona: Paidós Ibérica.

Bachelard, G. (2000). La poética del espacio. Buenos Aires: Fondo de Cultura Económica de Argentina S. A.

Bourdieu, P. (1991). Sobre la escuela. Entrevista recuperada el 10 de abril de 2011, disponible en www. youtube.com/watch?v=3mChkak7_3A

Castoriadis, C. (1986). Los dominios del hombre: encrucijadas del laberinto II. Argentina: Gedisa.

Guarín, G. (2004). Las crisis de la universidad contemporánea. Conferencia presentada en el Seminario de inducción a docentes de la Universidad La Gran Colombia, Julio, Armenia.

Luhmann, N. (1992). Sociología del riesgo. México: Universidad Iberoamericana, Publicaciones.

(1996). Teoría de la sociedad y pedagogía. Barcelona: Paidós Educador.

Lyotard, J. (2000). La condición postmoderna. Madrid: Cátedra Teorema.

Max-Neef, M. (1991). De la esterilidad de la certeza a la fecundidad de la incertidumbre. Ponencia presentada al Congreso internacional de la creatividad. Universidad Javeriana, facultad de psicología, Bogotá.
Mélich, J. (1998). Totalitarismo y Fecundidad. Monterrey: Anthropos Editorial.

Mitchell, J. (1994). Negociando los contextos de la prevención de desastres. Versión modificada de la ponencia presentada en el Seminario internacional Sociedad y prevención de desastres. COMECSO, UNAM, CONACYT, LA RED. Traducción de Elizabeth Mansilla e Ignacio Rubio, México.

Nietzsche, F. (2000). Sobre el porvenir de nuestras instituciones educativas. Barcelona: Tusquets.

Terren, E. (1999). Educación y modernidad: entre la utopía y la burocracia. Barcelona: Anthropos.

Ranciere, J. (2007). El maestro ignorante: cinco lecciones sobre la emancipación intelectual. Buenos Aires: Libros del Zorzal.

Vélez, B. (2006). Condiciones de posibilidad para la configuración de sentido en las comunidades de la Universidad. Tesis de maestría no publicada, Universidad de Manizales.

(2010). Universidad: sociedad, crisis y compromiso. Revista SOPHIA. VI edición, 11-21. Universidad La Gran Colombia, Armenia. 
